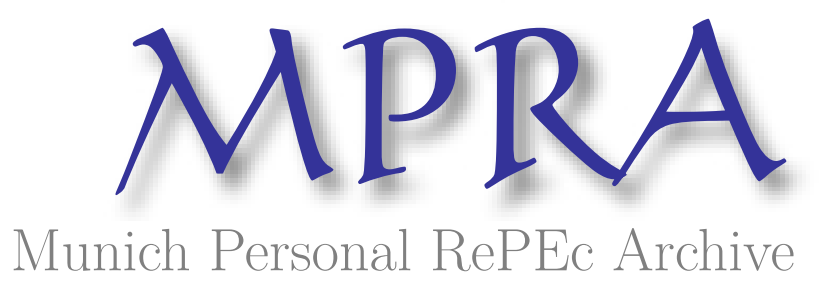

\begin{abstract}
A benchmarking model for measuring the efficiency of a humanitarian aid program: a case study of an international NGO.
\end{abstract}

Malki, Elli

22 October 2008

Online at https://mpra.ub.uni-muenchen.de/11222/

MPRA Paper No. 11222, posted 23 Oct 2008 05:53 UTC 


\title{
A benchmarking model for measuring the efficiency of a humanitarian aid program: a case study of an international NGO.
}

Elli Malki

Financial-Tip

POB 44034

Jerusalem 91440, Israel.

malki@netmedia.net.il

October 2008

\begin{abstract}
This article presents a benchmarking model for measuring the efficiency of organizations that provide humanitarian aid. The model was developed and implemented in the framework of a large international aid program. The model is based on measuring the labor productivity of each organization that provides services and comparing it to the benchmark. Two main results came out from the estimation of the model: (a) there were positive economies of scale in the program meaning that larger organizations were inherently more efficient than smaller ones; (b) the source of the inefficiency was identified, not in the administrative part of the organization, but rather in the programmatic part.
\end{abstract}

\section{1) Introduction}

The efficiency of NGOs has become a subject to an extensive research and a hot debate. On one hand foundations, donors and the general public are concerned about the way NGOs spend their money and want to ensure that they do it in the most cost effective manner. Trussel \& Parsons (2003) have shown that efficiency, measured by low overhead rate, is one of the main factors that influence the inflow of donations.

On the other hand the traditional focus on overhead rate is controversial. The recent Non Profit Overhead Cost Project, executed by the Center on Nonprofits and Philanthropy and the Urban Institute Center on Philanthropy in the Indiana University, concluded that NGOs effectiveness is damaged by the pressure to reduce overhead costs. This point is also advocated in a recent report by the Bridgespan Group, a Boston based consultancy that works with NGOs.

Why is this issue so important? Many NGOs are not exposed to the competitive environment that characterizes the for-profit sector. Competition imposes efficiency on commercial companies since less efficient companies will eventually, either be forced to improve, or will disappear. On the other hand NGOs that provide free, or highly subsidized services, have to compete for funding, but not for clients. Thus, inefficient NGOs will provide less (or lower quality) services, but their clients may not know it or will not have any other option. Since the market mechanism that enforces efficiency does not exist for NGOs, foundations and donors are rightfully concerned.

In this article I present a model for measuring efficiency, that was developed and implemented in a large NGO for its international aid program. In a previous article (Malki, 2008) I have presented this NGO, that I referred to as Charity-X, and a budgetary allocation model that was used by it for the same aid program.

Charity- $X$ is engaged in an extensive aid program that serves more than 200,000 clients in several countries. The target population of Charity- $X$ are the elderly, and the goal of the program is to bring the clients to an acceptable standard of living for the rest of their lives. Charity-X's program consists of a network of 170 welfare centers that are located in main cities of 9 countries. These centers provide eligible clients with basic material assistance like basic food products, canteen meals and basic medicines. About $12 \%$ of the clients have functional limitations and receive also home care services. The service model of each one of the centers is basically identical. 


\section{2) Oversight and efficiency measures}

Charity-X oversees these welfare centers with several computerized systems:

1. A budgetary and financial system that controls the use of budgets.

2. A management information system (MIS) that collects data on clients including their income level, functional status and a full documentation of the services that they received.

3. A procurement control and authorization system that is used for conducting tenders and overseeing purchases.

In addition to the above Charity-X decided to initiate several procedures to oversee the efficiency of the welfare centers. The need for such procedures rose as the increase in the local inflation rates diminished the level of services that were provided. Several efficiency measures were put into use:

\section{Overhead rates:}

In the process of approving the budgets of the welfare centers, Charity-X's management reviewed the overhead rate of each center. This process enabled Charity- $X$ to reduce administrative costs in a few locations, but did not bring significant efficiency gains. An in-depth analysis of the organizations with the highest overhead rates revealed that the reason for such rates, in most of the cases, was high rents paid for the facilities. While some of the welfare centers had a rent-free facility (either they owned it or received it from the local authorities for a free use), others had to pay full market rates. Thus, the review of overhead rates provided limited benefit in the attempt to improve efficiency.

\section{Cost per client:}

Since all the welfare centers provide an almost identical basket of services, the cost per client can serve as an indicator for efficiency. Lower cost per client represents a more efficient organization and vice versa. However, the comparison of cost per client is blurred because of the difference in price levels, both between countries and between large cities and peripheral locations. Since such differences in the level of prices were quite significant, it was not clear whether the cost per client is a reliable measure for efficiency.

\section{Productivity}

In order to overcome the problems of the cost per client, Charity-X tried an alternative measure the total number of clients divided by the number of full time equivalents (FTEs). This measure is usually called "labor productivity" or just "productivity". Staff is a major cost component of the welfare centers and the level of staffing was fully controlled by the centers managements. Unlike purchases that were controlled by Charity-X through its computerized procurement system there was no real oversight on the level of staffing. Productivity is a measure of efficiency that is not dependent on the price level and thus represents directly how efficiently each welfare center utilizes its staff.

\section{3) Data and methodology}

Charity-X surveyed all the 170 welfare centers and received data on the number of FTEs in each one of them. FTEs were calculated only from the permanent staff of each welfare center. The welfare centers also employed hourly employees mainly for the provision of home care services. These employees were no counted since it was not possible to calculate the number of FTEs that they represented.

The data on the number of clients was taken from Charity-X's MIS.

An overview of the data is presented in Table 1 herein: 
Table 1:

\begin{tabular}{|l|r|}
\hline \# of organizations & 170 \\
\hline Minimal \# of clients & 45 \\
\hline Maximal \# of clients & 25,247 \\
\hline Median \# of clients & 538 \\
\hline Minimal \# of FTEs & 1 \\
\hline Maximal \# of FTEs & 142 \\
\hline Median \# of FTEs & 8 \\
\hline Minimal productivity & 10.4 \\
\hline Maximal productivity & 832.2 \\
\hline Average productivity & 85.3 \\
\hline
\end{tabular}

The 170 welfare centers, supported by Charity-X, ranged from small peripheral centers (serving around 50 clients) to large mega-centers serving 15,000-25,000 clients. The median center serves about 540 clients.

The number of FTEs ranged from 1 to 142 and the median number of FTEs was 8 .

The productivity also varied from 10 to 832 with an average value of 85 .

In order to use the productivity as a measure for efficiency we need to understand its behavior. The functional relation between the number of clients and the productivity can be one of the three following forms:

- No economies of scale: an increase in the number of clients requires a similar increase in the number of FTEs. The productivity remains constant as the number of clients increases.

- Positive economies of scale: an increase in the number of clients requires a smaller increase in the number of FTEs. The productivity increases as the number of clients increases.

- Negative economies of scale (or diseconomies of scale): an increase in the number of clients requires a larger increase in the number of FTEs. The productivity decreases as the number of clients increases.

It is also possible that organizations will have positive economies of scale up to a certain size and then negative economies of scale. In this case the productivity rises with the increase in the number of clients, but only up to a certain point after which it starts to decrease.

In order to determine the functional relation between the productivity and the number of clients I have examined two alternative specifications: the logarithmic model and the trans logarithmic model.

The logarithmic model:

$$
\operatorname{Ln}(\text { Productivity })=\alpha+\beta * \operatorname{Ln}(\text { Clients })+\varepsilon
$$

Clients - the number of clients served by the organization.

Productivity - number of clients / number of FTEs.

The first derivative of equation (1) is the elasticity of the productivity in relation to the number of clients:

$$
\mathrm{dLn}(\text { Productivity }) / \mathrm{dLn}(\text { Clients })=\beta
$$

The interpretation of $\beta$ is that a $1 \%$ increase in the number of clients will induce a $\beta \%$ change in the productivity. 
Thus:

If $\beta>0$ there are positive economies of scale (productivity rises when the number of clients increases).

If $\beta<0$ there are negative economies of scale (productivity declines when the number of clients increases).

If $\beta=0$ there are no economies of scale (productivity is constant and is not related to the number of clients).

The logarithmic model can be a good approximation for the relation between the productivity and the number of clients as long as the elasticity is constant. However, there is no theoretical reason why the elasticity should be constant. In order to capture the possibility of a varying elasticity a more flexible model is required.

The trans logarithmic model:

(3) $\operatorname{Ln}($ Productivity $)=\alpha+\beta 1 * \operatorname{Ln}($ Clients $)+0.5 * \beta 2 *(\operatorname{Ln}(\text { Clients }))^{2}+\varepsilon$

The first derivative of equation (3) is:

$$
\mathrm{dLn}(\text { Productivity }) / \mathrm{dLn}(\text { Clients })=\beta 1+\beta 2 * \operatorname{Ln}(\text { Clients })
$$

The elasticity of the productivity in relation to the number of clients is not constant, but rather varies with the number of clients. When $\beta 2=0$ we return to the logarithmic model which is a special case of the trans logarithmic model. Regardless of the sign of $\beta 1$, as the number of clients grows the sign of the elasticity will depend on the sign of $\beta 2$.

If $\beta 2>0$ there will always be a large enough number of clients that will make the elasticity positive and eventually there will be positive economies of scale.

If $\beta 2<0$ the elasticity is bound to become negative, as the number of clients increases, and thus there will be eventually negative economies of scale.

\section{4) The results}

The aforementioned specifications were estimated using OLS regressions. Table 2 herein presents the results of the logarithmic model.

Table 2: Logarithmic model

\begin{tabular}{|l|c|c|}
\hline \multicolumn{2}{|l|}{ Regression Results - OLS } & \\
\hline $\begin{array}{l}\text { Number of } \\
\text { observations }\end{array}$ & 170 & \\
\hline R Square & 0.541 & \\
\hline Adjusted R square & 0.538 & \\
\hline SE & 0.423 & \\
\hline F statistic & 197.8 & \\
\hline & & \\
\hline & $\mathrm{a}$ & $\mathrm{b}$ \\
\hline Coefficient & 1.733 & 0.383 \\
\hline SE coefficient & 0.179 & 0.027 \\
\hline t statistic & 9.696 & 14.064 \\
\hline Regression Results - WLS & \\
\hline \multicolumn{2}{|c|}{ W } & $\mathrm{a}$ \\
\hline Coefficient & 1.631 & 0.398 \\
\hline SE coefficient & 0.169 & 0.027 \\
\hline t statistic & 9.673 & 14.484 \\
\hline
\end{tabular}


The overall $\mathrm{F}$ statistic of the regression is high and the two estimated coefficients are significantly different from zero. Overall the model is able to explain about $54 \%$ of the variation in the data. The fact that $b>0$ and is significant suggests that there are positive economies of scale. The point estimator of the elasticity of the productivity in relation to the number of clients is 0.383 . Thus a $1 \%$ increase in the number of clients will induce $0.38 \%$ increase in the productivity.

One pitfall in the analysis is that cross sectional data are, in many cases, heteroscedastic. Thus, I have preformed a Goldfeld-Quandt test using the first 60 observations and the last 60 observations. The F statistic was 2.184 which is high enough to reject the null hypothesis of homoscedasticity. The test suggests that when the number of clients increases, the variance of the residuals rises. In this case the OLS estimators are unbiased but inefficient. Thus I have computed a WLS regression using the variable $\mathrm{Ln}$ (Clients) as the weighting factor. The results are presented in the second part of Table 2. The change is the coefficients' estimators is quite modest. The new point estimator for the elasticity of the productivity in relation to the number of clients is slightly higher -0.398 . A graphical presentation of the results of the logarithmic model (equation 1), using the WLS estimators, is depicted in figure 1 hereinafter (the values on the $\mathrm{X}$ axis and $\mathrm{Y}$ axis are in natural logarithms).

\section{Figure 1: Logarithmic model}

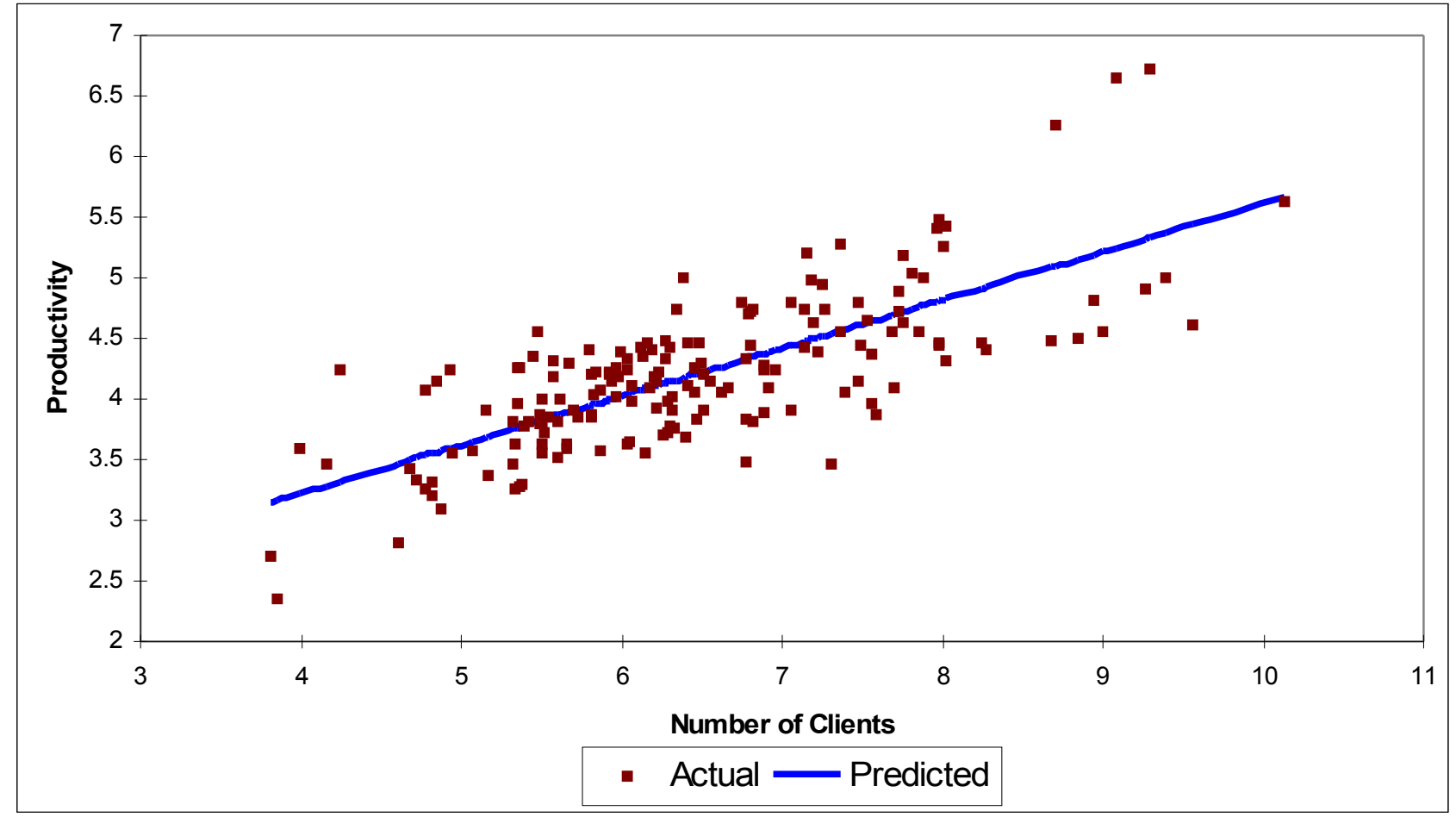

The straight line represents the predicted value of the productivity, based on the logarithmic model. The linearity is a the result of our assumption that the elasticity is constant, but the positive relation between the productivity and the number of clients is evident from the scatter plot. The points along the straight line represent the expected productivity as a function of the number of clients. As the number of clients increases we expect also a higher productivity. The points of the scatter plot above the line represent organizations with higher than the norm productivity. These organizations are able to utilize their labor more effectively. The points below the line represent the less efficient organizations which are over-staffed.

Table 3-a presents the OLS and WLS estimators of the trans logarithmic model (WLS was computed in the same manner as in the logarithmic model by using the variable Ln(Clients) as the 
weighting factor).

Table 3-a: Trans logarithmic model

\begin{tabular}{|l|c|c|c|}
\hline $\begin{array}{l}\text { Regression Re sults - OLS } \\
\text { Number of } \\
\text { observations }\end{array}$ & 170 & & \\
\hline R Square & 0.546 & & \\
\hline Adjusted R square & 0.540 & & \\
\hline SE & 0.424 & & \\
\hline F statistic & 100.3 & & \\
\hline & & & $b 2$ \\
\hline & $\mathrm{a}$ & $\mathrm{b} 1$ & -0.012 \\
\hline Coefficient & 1.444 & 0.467 & 0.033 \\
\hline SE coefficient & 0.745 & 0.223 & -0.364 \\
\hline $\mathrm{t}$ statistic & 1.938 & 2.091 & $\mathrm{~b} 2$ \\
\hline Regression Re sults - WLS & & -0.033 \\
\hline & $\mathrm{a}$ & $\mathrm{b} 1$ & 0.032 \\
\hline Coefficient & 0.995 & 0.607 & -1.018 \\
\hline SE coefficient & 0.647 & 0.207 & \\
\hline t statistic & 1.538 & 2.936 & \\
\hline
\end{tabular}

The trans logarithmic model fits the data worse than the logarithmic model. The estimator b2 (which estimates $\beta 2$ ) is negative, which means negative economies of scale, but is not significantly different from zero. The estimator of the intercept a, in the WLS regression, is also not significantly different from zero. Thus, it seems that the logarithmic model, which implies positive economies of scale with constant elasticity, describes the data more accurately.

A second version of the trans logarithmic model was tested with the restriction that the constant is zero. The existence of a constant term in equation (3) is not derived from the economic theory and it is an empirical question. Since the estimator of the constant term in the WLS regression was not significant it makes sense to estimate the regression without it. The results of the OLS and WLS regressions without the constant term are presented in Table 3-b.

Table 3-b: Trans logarithmic model with no constant

\begin{tabular}{|l|c|c|c|}
\hline Regression Re sults - OLS & & \\
\hline $\begin{array}{l}\text { Number of } \\
\text { observations }\end{array}$ & 170 & & \\
\hline R Square & 0.990 & & \\
\hline Adjusted R square & 0.984 & & \\
\hline SE & 0.427 & & \\
\hline F statistic & 8303.4 & & \\
\hline & & & $b 2$ \\
\hline & $a$ & $b 1$ & -0.074 \\
\hline Coefficient & 0 & 0.896 & 0.008 \\
\hline SE coefficient & & 0.028 & -9.270 \\
\hline$t$ statistic & & 32.156 & $b 2$ \\
\hline Regression Re sults - WLS & & -0.081 \\
\hline & $a$ & $b 1$ & 0.008 \\
\hline Coefficient & 0 & 0.921 & -9.566 \\
\hline SE coefficient & & 0.028 & \\
\hline$t$ statistic & & 33.079 & \\
\hline
\end{tabular}

The two coefficients b1 and b2 are highly significant and the negative sign of b2 implies that there 
are negative economies of scale. The $\mathrm{R}$ square and the adjusted $\mathrm{R}$ square are very high, however the exclusion of the constant term blurs the meaning of the $\mathrm{R}$ square as a measure of goodness of fit since it is not bound between 0 and 1. Figure 2 presents a visual comparison of the goodness of fit of the trans logarithmic model (model 2) versus the logarithmic model (model 1). It is not possible to judge, based on the graph, which model fits the data more accurately, but we cannot exclude the possibility that the correct specification is the trans logarithmic model.

The trans logarithmic model suggests negative economies of scale: the elasticity of the productivity in relation to the number of clients decreases as the number of clients increases and eventually will become negative. However, within the range of our dataset the elasticity remains positive. A $1 \%$ increase in the number of clients will induce a $0.61 \%$ increase in productivity in the smallest organization (45 clients), a $0.41 \%$ increase in productivity in the median organization (540 clients) and a $0.1 \%$ increase in the largest organization (25,000 clients). Thus we can see that in the 170 organizations that were examined, positive economies of scale exist regardless of the model that we choose (the point where the elasticity changes its sign is reached when the number of clients exceeds 85,000).

As we can see the two regressions do not provide a conclusive evidence which one of the models has a better predictive power. Nevertheless, there are two reasons that make the trans logarithmic model more desirable:

- The first reason is that the implication of logarithmic model is that the positive economies of scale are always there, no matter how large the organization becomes. This result is in contradiction with the practical experience that when an organization becomes too large it will be less efficient mainly due to scarce managerial capacity. The trans logarithmic model seems to be more in line with the real life experience.

- The second reason is that we are looking for the outliers that are far below the line of the predicted values. These outliers represent organizations that will have to cut staff based on the predictions of the model. Since the trans logarithmic model is convex, using it will result in a more lenient decision on the level of staff reduction that is needed. This seems as a more desirable approach given the severity of the decisions.

Figure 2: Comparison between the models

(the values on the $\mathrm{X}$ axis and $\mathrm{Y}$ axis are in natural logarithms)

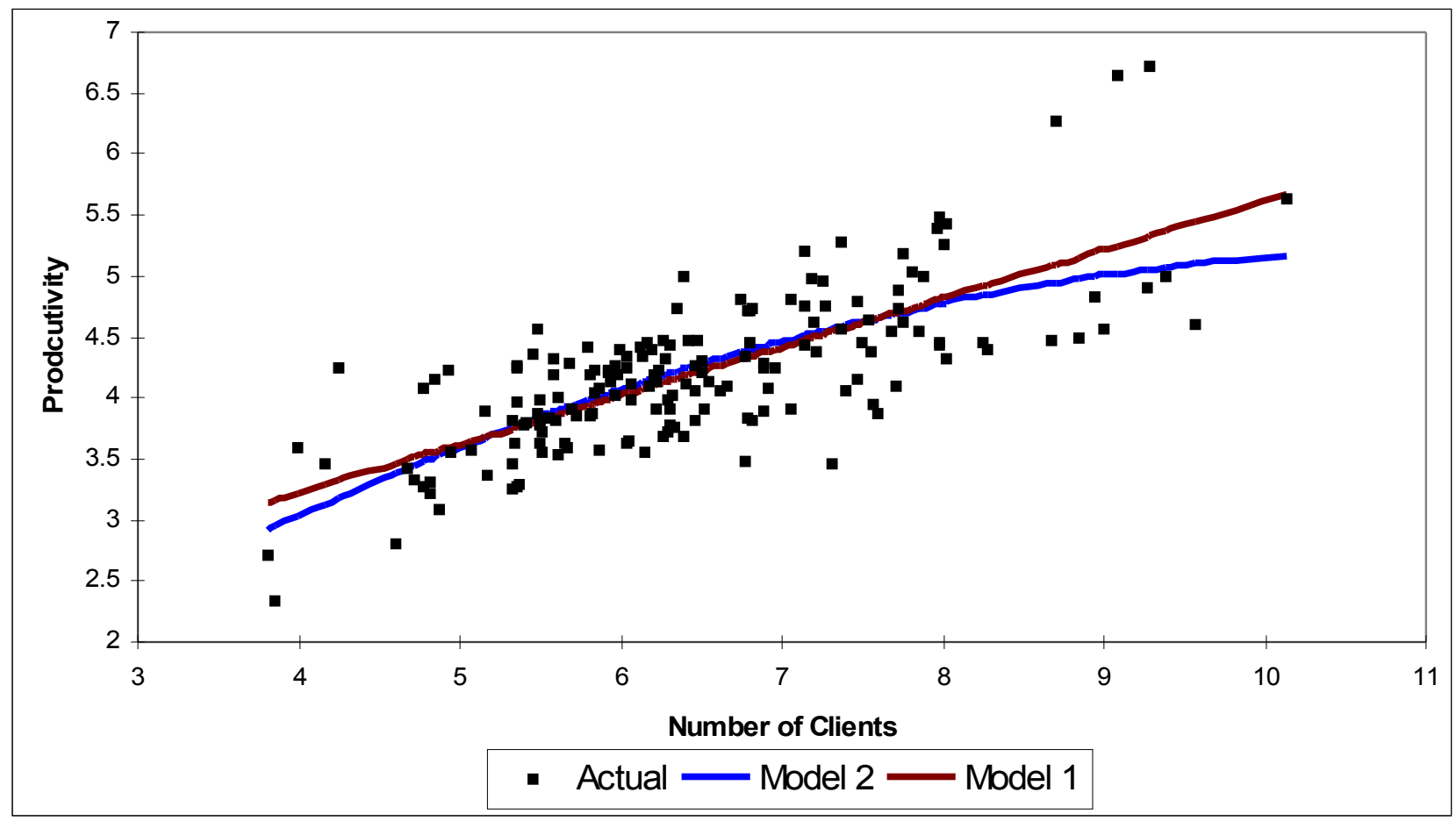


Thus, all the results that are presented hereinafter were calculated using the trans logarithmic model. Nevertheless, for practical application the logarithmic model can be used as well with little difference in the results.

The only significant difference between the models is the conclusion about the optimal size of the welfare organization. The conclusion from the logarithmic model is that the larger is the better. It is always worthwhile to encourage small organizations to merge and become a larger organization. The conclusion from the trans logarithmic model is that size has benefits only up to a certain point. When the number of client exceeds 10,000 the increase in productivity, which results from $1 \%$ increase in the number of clients, is merely $0.17 \%$. Since merging organizations involves costs that are not accounted for in this simple model, the outcome may be too small to justify the effort. The results of the regression suggest that, of the 170 organizations supported by Charity-X, 89 (52.4\%) have productivity which is lower than the norm and thus are over-staffed. An interesting question is whether there is a connection between over-staffing and the size of the organization. In order to examine this issue the organizations were divided to three groups: small organizations (less than 1,000 clients), medium organizations (between 1,000 to 3,000 clients) and large organizations (above 3,000 clients). The results are presented in Table 4.

Table 4: Over/Under staffing by size

\begin{tabular}{|l|c|c|c|c|}
\hline & $\begin{array}{c}\text { Small } \\
\text { organizations }\end{array}$ & $\begin{array}{c}\text { Medium } \\
\text { organizations }\end{array}$ & $\begin{array}{c}\text { Large } \\
\text { organizations }\end{array}$ & Total \\
\hline \# of organizations & 118 & 36 & 16 & 170 \\
\hline \# of over staffed & 61 & 18 & 10 & 89 \\
\hline$\%$ of over staffed & $51.7 \%$ & $50.0 \%$ & $62.5 \%$ & $52.4 \%$ \\
\hline
\end{tabular}

We can see that there is no connection between over staffing and size in the small and the medium organizations, which are the majority. In both groups there is an almost equal division between over-staffed and under staffed organizations. However in the group of the large organizations the proportion of the over-staffed organizations is higher $-62.5 \%$. Although this group is the smallest and represents only $10 \%$ of the organizations, in budgetary terms it is the most significant group that receives about $50 \%$ of the program's budget. Thus, the results suggest that too many large organizations did not manage to exploit their economies of scale and thus were less efficient.

\section{5) Additional analysis}

The aforementioned model provides a tool to improve the efficiency of the welfare organizations by reducing the staff level. For each organization the normative staff level was calculated, using the predicted values from model (3). The calculation of the normative staff level was done as follows:

(5) Normative \# of FTEs $=$ Clients / $\mathrm{e}^{\mathrm{y}}$

$y-$ the predicted value from the estimation of equation (3).

Large number of actual of FTEs compared to the predicted number of FTEs means that the productivity of the organization is low and there is a need to reduce staff.

A further insight into the source of inefficiency was obtained by comparing the rate of over/under staffing to the cost data of a sample of 13 welfare organizations. These organizations were chosen judgmentally to represent the program's budget. 7 of them are large organizations (above 3,000 clients), representing $44 \%$ of this group and 5 are medium organizations representing $14 \%$ of the this group. In terms of geographical representation 11 of the 13 organizations are located in the two countries in which $80 \%$ of program's budget is invested. The other 2 organizations are the largest in 
other countries that are covered by the program. Thus the sample of the 13 organizations provides an informative representation of the major part of the program's budget. For each one of the organizations I have calculated the total cost per client, program's cost per client and overhead cost per client. These figures were compared to the rate of over/under staffing and the coefficients of correlation were calculated. The results are presented in Table 5 herein (financial data are on annual basis and relate to the year 2006).

Table 5:

\begin{tabular}{|l|c|c|c|c|c|}
\hline Organization & \# of Clients & $\begin{array}{c}\text { Total cost } \\
\text { per client (\$) }\end{array}$ & $\begin{array}{c}\text { Program's } \\
\text { cost per } \\
\text { client (\$) }\end{array}$ & $\begin{array}{c}\text { Overhead } \\
\text { cost per } \\
\text { client (\$) }\end{array}$ & $\begin{array}{c}\text { Over/Under } \\
\text { staffing in } \\
\%\end{array}$ \\
\hline Org. 1 & & & & & \\
\hline Org. 2 & 666 & 241.8 & 181.5 & 60.3 & $\mathbf{0 . 0 \%}$ \\
\hline Org. 3 & 2,459 & 127.2 & 94.5 & 32.7 & $\mathbf{- 3 7 . 5 \%}$ \\
\hline Org. 4 & 2,873 & 207.3 & 176.9 & 30.4 & $\mathbf{- 9 2 . 3 \%}$ \\
\hline Org. 5 & 2,909 & 102.5 & 87.5 & 15.0 & $\mathbf{- 1 0 8 . 3 \%}$ \\
\hline Org. 6 & 2,933 & 244.1 & 222.2 & 21.9 & $\mathbf{2 6 . 5 \%}$ \\
\hline Org. 7 & 3,033 & 261.7 & 220.9 & 40.7 & $\mathbf{3 8 . 3 \%}$ \\
\hline Org. 8 & 6,915 & 235.4 & 213.2 & 22.2 & $\mathbf{3 7 . 7 \%}$ \\
\hline Org. 9 & 7,699 & 140.5 & 116.0 & 24.4 & $\mathbf{1 6 . 1 \%}$ \\
\hline Org. 10 & 8,156 & 252.1 & 227.2 & 24.9 & $35.7 \%$ \\
\hline Org. 11 & 10,573 & 246.8 & 221.1 & 25.7 & $\mathbf{1 3 . 4 \%}$ \\
\hline Org. 12 & 14,245 & 247.2 & 225.2 & 22.1 & $\mathbf{3 8 . 7 \%}$ \\
\hline Org. 13 & 25,247 & 135.2 & 115.9 & 19.3 & $\mathbf{- 5 8 . 9 \%}$ \\
\hline Correlation & & & & & \\
with & & & & & \\
Over/Under & -0.02 & $\mathbf{0 . 7 3}$ & $\mathbf{0 . 7 4}$ & $\mathbf{0 . 1 7}$ & \\
\hline Staffing & & & & & \\
\hline
\end{tabular}

The right column of the table is the rate of over/under staffing, which is the difference in percentage terms between that actual and the normative number of FTEs. A positive figure means that the number of actual FTEs is higher than the normative number of FTEs which means low efficiency. The last row in the table presents the coefficients of correlation between the rate of over/under staffing and the other variables.

There is no correlation between the rate of over/under staffing and the number of clients. This result is in accordance with the results that were already presented before (see Table 4).

The correlation between the rate of over/under staffing and the total cost per client is positive and high (0.73). However, this correlation stems from the high correlation between the program's cost per client and the rate of over/under staffing (0.74). There is almost no correlation between the overhead cost per client and the rate of over/under staffing $(0.17)$.

Figure 3 depicts the correlation between the rate of over/under staffing and the programmatic cost per client in the sample of the 13 organizations. 
Figure 3:

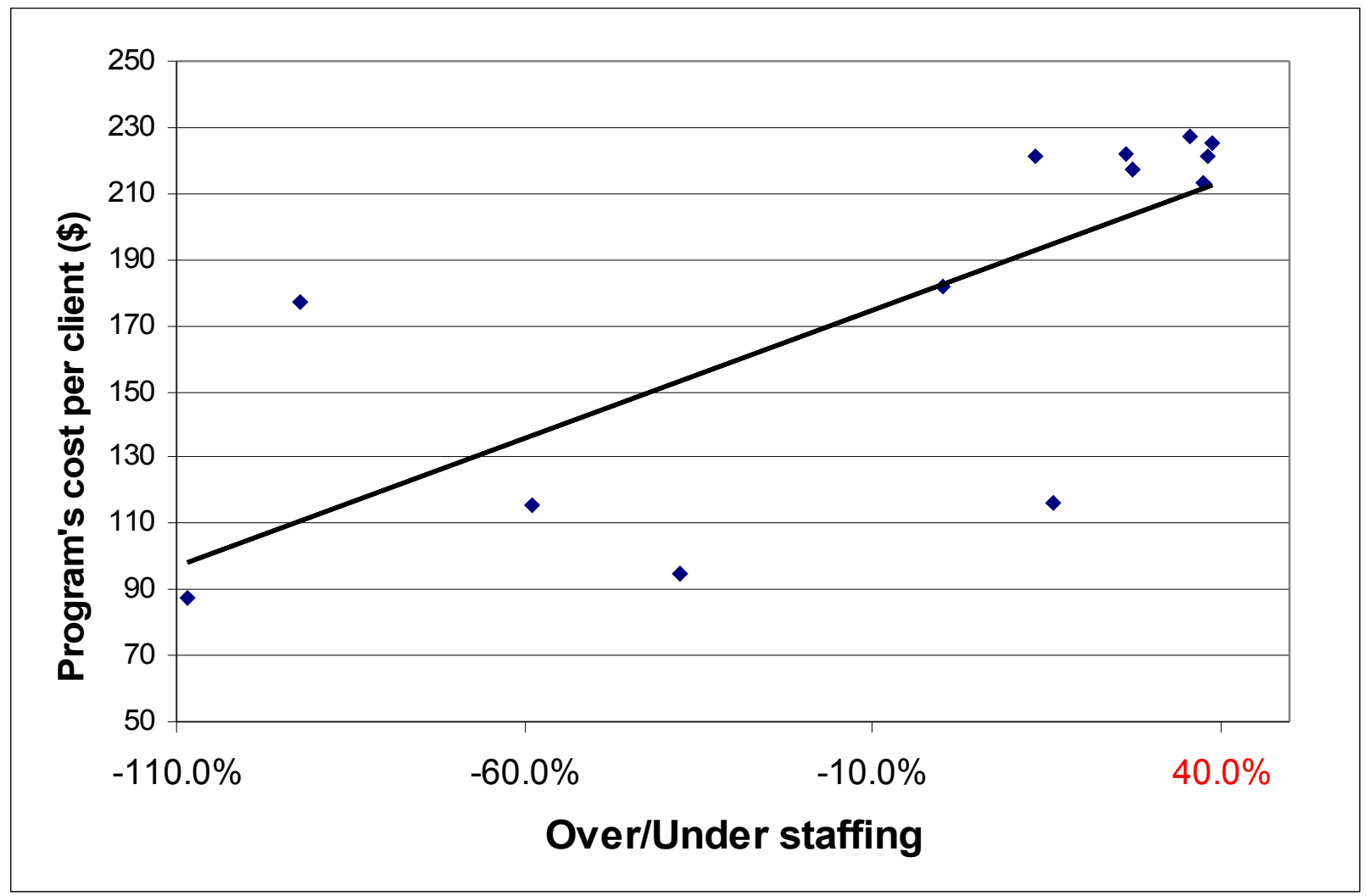

This is an interesting finding since it does not conform to the common practice of attributing inefficiencies to the overhead expenses. Evidently, it's the programmatic staff that suffers from the lack of productivity and should be reduced. This conclusion is not unique to this study; similar results were reported by Bradley, Jansen and Silverman (2003). The aforementioned results support the criticism on the focus on overhead rate as a primary measure of inefficiency. At the same time they demonstrate that high rate of programmatic cost does not necessarily imply that the organization is doing well.

\section{6) Conclusions}

The evaluation of their efficiency is a central issue for NGOs. Donors, foundations and governmental agencies are putting strong emphasis on efficiency, but usually use only the traditional measures that are related to the overhead and administrative expenses. This article presents an alternative model that focuses on the programmatic part of the expenses. On top of the proposed model, that can be used by other NGOs, there are two conclusions, that came out of this study that are more general relevancy:

- The results show the existence of positive economies of scale in the humanitarian aid program, regardless of the model that was used for estimation. This is an important observation; positive economies of scale mean that smaller organizations are inherently less efficient than larger ones. Merging is not very acceptable in the third sector, but it may desirable in order to increase efficiency.

- The source of the inefficiency was identified, not in the administrative part of the organization, but rather in the programmatic part. The focus on the reduction of overhead rates is misleading and may even cause damage to NGOs, as other studies suggest. Measures that focus on the execution of the program, like program's cost per client or labor productivity, seems to be more reliable and effective. 


\section{7) References}

Bedsworth W., Goggins Gregory A., \& Howard D. (2008), Nonprofit Overhead Costs: Breaking the Vicious Cycle of Misleading Reporting, Unrealistic Expectations, and Pressure to Conform, The Bridgespan Group, Inc. (April).

http://www.bridgespan.org/kno_articles npoverheadcosts.html

Bradley B., Jansen P. \& Silverman L. (2003), The Nonprofit Sector's \$100 Billion Opportunity, Harvard Business Review, (May), pp 94-103.

Greene W.H. (1990), Econometric Analysis, Macmillan Publishing Company.

Malki E. (2008), Systematic Approach to the Allocation of Budgets for Humanitarian Aid Programs: A Practical Tool for International NGOs, SSRN.

http://papers.ssrn.com/sol3/papers.cfm?abstract id=1266334

Nonprofit Overhead Cost Project; Center on Nonprofits and Philanthropy, Urban Institute Center on Philanthropy, Indiana University (2004), What We Know About Overhead Costs in the Nonprofit Sector: Research Brief \#1.

Getting What We Pay For: Low Overhead Limits Nonprofit Effectiveness: Research Brief \#3. The Pros and Cons of Financial Efficiency Standards: Research Brief \#5. http://nccsdataweb.urban.org/FAQ/index.php?category=51\#521

Pindyck R.S. \& Rubinfeld D.L. (1981), Econometric Models and Economic Forecasts, McGraw Hill Book Company.

Pollack T.H. \& Rooney P. (2003), Management and General Expenses: The Other Half of Overhead, The Nonprofit Quarterly, (Spring), pp 30-32.

Trussel J. M. \& Parsons L. M. (2003), Financial Reporting Factors Affecting Donations to Charitable Not-for-Profit Organizations, SSRN.

http://papers.ssrn.com/sol3/papers.cfm?abstract id $=481383$ 